\title{
Analysis of the proton longitudinal structure function from the gluon distribution function
}

\author{
G.R.Boroun* and B.Rezaeit \\ Physics Department, Razi University, Kermanshah 67149, Iran
}

(Dated: October 26, 2018)

\begin{abstract}
We make a critical, next- to- leading order, study of the relationship between the longitudinal structure function $F_{L}$ and the gluon distribution proposed in Ref.[2], which is frequently used to extract the gluon distribution from the proton longitudinal structure function at small $x$. The gluon density is obtained by expanding at particular choices of the point of expansion and compared with the hard pomeron behaviour for the gluon density. Comparisons with H1 data are made and predictions for the proposed best approach are also provided.
\end{abstract}

\section{Introduction}

The longitudinal proton structure function $F_{L}$, measured in the deep inelastic lepton- proton scattering, is proportional to the cross section for the interaction of the longitudinally polarized virtual photon with a proton. This observable is of particular interest since it is directly sensitive to the gluon density. In the quarkparton model, the longitudinal structure function is zero since longitudinal polarized photons do not couple to spin $\frac{1}{2}$ quarks. In the lowest order DGLAP [1] approximation of $\mathrm{QCD}$, the longitudinal structure function is given by

$$
F_{L}\left(x, Q^{2}\right)=K^{S+N S} \otimes F_{2}\left(x, Q^{2}\right)+K^{G} \otimes G\left(x, Q^{2}\right),
$$

where both quarks and gluons contribute. The variable $Q^{2}$ is the square of the four- vector momentum exchange, and $x$ is the Bjorken scaling variable. Here kernels $\left(K^{i}\right)$ are the coefficients functions and the symbol $\otimes$ denotes convolution according to the usual prescription. At small values of $x, F_{L}$ is driven mainly by gluons through the transition $g \rightarrow q \bar{q}\left(g\left(x, Q^{2}\right)\right.$ is the gluon density). Therefore $F_{L}$ can be used for the extraction of the gluon distribution in the proton, and it provides a crucial test of the validity of perturbative QCD in this kinematical range. The knowledge of $F_{L}$ gives a complementary determination of the gluon distribution to that using the scaling violation of $F_{2}$, since at low $x$ we have

$$
F_{L}^{g}\left(x, Q^{2}\right)=K_{L O+N L O}^{G} \otimes G\left(x, Q^{2}\right) .
$$

In this paper we deduce the general relations between the longitudinal structure function and the gluon distribution function with analytical methods at NLO. In Ref.[2] a relationship between $F_{L}$ and the gluon was proposed in order to facilitate the extraction of the gluon density from data. We demonstrate here

\footnotetext{
*Electronic address: grboroun@gmail.com; boroun@razi.ac.ir

†brezaei@razi.ac.ir
}

that the validity of this relation crucially depends on the choice of expansion point. Our purpose here is to improve the situation with a new expansion of the gluon distribution function at LO and NLO. In addition our results provide a useful NLO expression valid at small $x$, where the gluon has a hard- Pomeron behaviour. Section 2 is devoted to a revision of the method proposed in Ref.[2] and gives a general expression at NLO. Finally, in section 3, we present our conclusions.

\section{Compact Formula}

The standard collinear factorization formula for the longitudinal structure function at low $x$ reads

$F_{L}^{g}\left(x, Q^{2}\right)=\int_{0}^{1-x} \frac{d z}{1-z} K_{L O+N L O}^{G}(1-z) G\left(\frac{x}{1-z}, Q^{2}\right)$,

where $F_{L}$ is derived from the integrated gluon distribution. The analytical expression of the gluon kernel [3-4] at LO and NLO is defined in the Appendix. An approximate relationship between $F_{L}$ and the gluon distribution can be derived from the expansion of $G\left(y, Q^{2}\right)$ around a chosen expansion point. Therefore, the choice of the point of expansion is associated with the relation between of $x_{g}$ and $x$.

A): In Ref.2 the authors have suggested that expression (3) at LO can be reasonably approximated by

$$
F_{L}^{g}\left(x, Q^{2}\right)=\frac{2 \alpha_{s}}{\pi} \frac{\sum_{i=1}^{N_{f}} e_{i}^{2}}{5.9} G\left(2.5 x, Q^{2}\right)
$$

which demonstrates the close relation between the longitudinal structure function and the gluon distribution at small $x$.

B): Using the expansion method for the gluon distri- 
bution function at an arbitrary point $z=a$ as

$$
\left.\frac{x}{1-z}\right|_{z=a}=\frac{x}{1-a} \sum_{k=1}^{\infty}\left[1+\frac{(z-a)^{k}}{(1-a)^{k}}\right] \text {. }
$$

The above series is convergent for $|z-a|<1$. According to this expression we can expand the gluon distribution $G\left(\frac{x}{1-z}\right)$ as

$$
\begin{aligned}
\left.G\left(\frac{x}{1-z}\right)\right|_{z=a}= & G\left(\frac{x}{1-a}\right) \\
& +\frac{x}{1-a}(z-a) \frac{\partial G\left(\frac{x}{1-a}\right)}{\partial x}+O(z-a)^{2} .
\end{aligned}
$$

Retaining terms only up to the first derivative in the expansion and doing the integration, we obtain our master formula as

$$
F_{L}^{g}\left(x, Q^{2}\right)=A\left(x, Q^{2}\right) \times G\left(\frac{x}{1-a}\left(1-a+\frac{B\left(x, Q^{2}\right)}{A\left(x, Q^{2}\right)}\right)\right),
$$

where

$$
A\left(x, Q^{2}\right)=\int_{0}^{1-x} \frac{1}{1-z} K_{L O+N L O}^{G}(1-z) d z
$$

and

$$
B\left(x, Q^{2}\right)=\int_{0}^{1-x} \frac{z-a}{1-z} K_{L O+N L O}^{G}(1-z) d z,
$$

where $\alpha$ has an arbitrary value $0 \leq \alpha<1$.

Eq.7 can be rewritten as

$$
F_{L}^{g}\left(x, Q^{2}\right)=\frac{10 \alpha_{s}}{27 \pi} G\left(\frac{x}{1-a}\left(\frac{3}{2}-a\right)\right)
$$

In the limit $x \rightarrow 0$ for LO analysis. This result shows that the longitudinal structure function $F_{L}^{g}\left(x, Q^{2}\right)$ at $x$ is calculated using the gluon distribution $\frac{x}{1-a}\left(\frac{3}{2}-a\right)$ at the limit $x \rightarrow 0$. When the points $a=0, a=0.666$ and $a=0.9$ are used, we get respectively

$$
\begin{gathered}
F_{L}^{g}\left(x, Q^{2}\right)=\frac{10 \alpha_{s}}{27 \pi} G\left(\frac{3}{2} x\right)(<\operatorname{Ref} \cdot[2]), \\
F_{L}^{g}\left(x, Q^{2}\right)=\frac{10 \alpha_{s}}{27 \pi} G(2.5 x)(=\operatorname{Ref} .[2]),
\end{gathered}
$$

and

$$
F_{L}^{g}\left(x, Q^{2}\right)=\frac{10 \alpha_{s}}{27 \pi} G(6 x)(>\operatorname{Ref} .[2]) .
$$

Therefore these equations show that our approximation differs from the result of Ref.[2] . In the expanding the gluon distribution around $z=0$, the result (11) differs from Ref.[2] result. We explain that difference arises in the expansion method. Our results cover expanding of the gluon distribution at all points of the expansion.
In Fig.1 we present the results of the longitudinal structure function at some points of expansion $z=a$ using the gluon distribution, which is usually taken from the Block [5] model at LO. The DL [6-7] fit and GRV [8] parameterizations have also been used to investigate quantitatively the accuracy of $F_{L}$ with respect to the gluon distribution. We introduced the fractional accuracy variable

$$
\text { Fractional Accuracy } \equiv F_{i, M R S T} \equiv 1-\frac{F_{L}(i)}{F_{L}(M R S T)}
$$

where $i=G R V, D L$ and Block distributions with $F_{L}(M R S T)$ denoting the predictions of the longitudinal structure function in NLO PQCD which are given by the MRST parametrizations [11]. We show in Fig.2 that the fractional accuracy for the the Block model is the best which confirms the correctness of our solution for $F_{L}$ with Block model. We also note that there is disagreement between Block distribution and NLO-QCD fit to $\mathrm{H} 1$ data. It is clear that Block starting distribution is at LO when compared with $\mathrm{H} 1$ data in an NLO analysis.

Therefore, in Fig.1, we compare our results at two points of the expansion $a=0$ and $a=0.9$ with the the gluon distribution input according to the Block model [5]. We compared our results with the results of Ref.[2], H1 data [9] and MVV prediction [10]. These results show that the expansion of the gluon distribution around $a=0$ differs from the Ref.[2] result, and is equal to that result only at the point of expansion $a=0.666$. But the best results can be found in the range of expansion at the points of $0.7 \leq a \leq 0.95$. It is easy to see that the validity of the expansion in LO depends strongly on the point of the expansion of the gluon distribution and this is comparable with the experimental data only at high values of the point of expansion.

We also obtain the relation between the longitudinal structure function and gluon distribution exact to NLO analysis which adopts the similar integral form (Eqs.710 ), where the coefficient function $K^{G}$ is well known up to NLO. Therefore, an explicit relation between the functions can be found at this order. Unfortunately there is not an analytical form at this order. Consequently, in order to estimate the magnitude of the NLO corrections for the low $x$ range, we have numerically computed $F_{L}^{g}\left(x, Q^{2}\right)$ at a moderate $Q^{2}=20 G e V^{2}$.

In order to show this we firstly computed the relation between $x_{g}\left(=k x, k=\frac{1}{1-a}\left(1-a+\frac{B}{A}\right)\right)$ and $x$ for three different of $x$ values at $Q^{2}=20 \mathrm{GeV}^{2}$ in Fig.3. These distributions represent the whole spectrum of possible behaviours of $x_{g}$ with respect to the points of the expansion at small $x$. They tend to constant values at small $x$ at NLO . Having done that we determined the longitudinal structure function at point of expansion $a=0$ and the point of expansion which corresponds 
to the maximal deviation from the result for $a=0$ for several chosen $x$ values. Since these latter points of expansion differ they are separately labelled on the Fig.4.

The results of this analysis are shown in Fig.4. We plot there the lower and higher limit of the point of expansion at $Q^{2}=20 \mathrm{GeV}^{2}$ in NLO analysis. It is easy to see that the validity of relation (7) in NLO depends strongly on the lower limit of the point of expansion. By contrast for the NLO analysis, the relation is still approximately true for the points of expansion bigger than 0.7. We can conclude that, when compared with the behaviour at LO, we cannot find a monotonic relation between $x_{g}$ and $x$ at NLO but comparison to $\mathrm{H} 1$ data suggests that the expansion point $a=0$ yields the best results.

C): Exploiting the low- $x$ behaviour of the gluon distribution function according to the hard (Lipatov) Pomeron as

$$
G\left(x, Q^{2}\right)=f\left(Q^{2}\right) x^{-\delta} .
$$

The power of $\delta$ is found to be either $\delta \simeq 0$ or $\delta \simeq 0.5$. The first value corresponds to the soft Pomeron and the second value to the hard (Lipatov) Pomeron intercept. Based on the hard (Lipatov) pomeron behaviour for the gluon distribution, let us put Eq.(15) in Eq.(3). After doing the integration over $z$, Eq.(3) can be rewritten as

$$
F_{L}^{G}\left(x, Q^{2}\right)=I_{G}\left(x, Q^{2}\right) \times G\left(x, Q^{2}\right),
$$

where

$$
I_{G}\left(x, Q^{2}\right)=\int_{0}^{1-x} k_{L O+N L O}^{G}(1-z)(1-z)^{\delta-1} d z .
$$

We observe that equation 16 implies a relationship between $F_{L}$ and the gluon at the same value of $x$.

In Fig.5 we present results for $F_{L}$ at LO and NLO using the gluon distribution [5]. This seems to indicate that $F_{L}$ is dominated at small $x$ by hard Pomeron exchange. This powerful approach to the small- $x$ data for $F_{L}^{g}\left(x, Q^{2}\right)$ extends the Regge phenomenology that is so successful for hadronic processes. The Ref.[2] result (solid line) is also shown. We immediately see that our results at LO and NLO with respect to the hard Pomeron behaviour have much better behaviour than the Ref.[2] result.

\section{Conclusions}

In this work we discuss the approximative determination of the longitudinal structure function at low $x$ with respect to the expansion of the gluon distribution at arbitrary point of expansion of $G\left(\frac{x}{1-z}\right)$ at LO and NLO. When comparing results, we conclude that the more suitable points of expansion are in the range $0.7 \leq a \leq 0.95$ at LO and $a \simeq 0$ at NLO. We demonstrate that the LO relation (4) between the longitudinal structure function and the gluon distribution is not generally valid. It remains a reasonable approximation only at the point of expansion $a=0.666$ for a gluon distribution which is sufficiently singular. At NLO the H1 data indicate that the optimal point of expansion is $a \simeq 0$. Fortunately, the hard Pomeron behaviour for the gluon distribution estimates $F_{L}$ at LO and NLO with reasonable validity for $x_{g}=x$.

Acknowledgements G.R.Boroun would like to thank the anonymous referee of the paper for his/her careful reading of the manuscript and for the productive discussions. 


\section{Appendix}

The explicit form of the gluon kernel is given by the following:

$$
\begin{aligned}
K_{L O}^{G}\left(\frac{x}{y}, Q^{2}\right)= & \frac{\alpha_{s}}{4 \pi}\left[8(x / y)^{2}(1-x / y)\right]\left[\sum_{i=1}^{N_{f}} e_{i}^{2}\right] \\
K_{N L O}^{G}\left(\frac{x}{y}, Q^{2}\right)= & \left(\frac{\alpha_{s}}{4 \pi}\right)^{2}\left[\sum_{i=1}^{N_{f}} e_{i}^{2}\right]\left[16 C_{A}(x / y)^{2}(+4 \operatorname{dil} \log (1-x / y)\right. \\
& -2(1-x / y) \ln (x / y) \ln (1-x / y)+2(1+x / y) \operatorname{dilog}(1+x / y)+3 \ln (x / y)^{2} \\
& +2(x / y-2) P i^{2} / 6+(1-x / y) \ln (1-x / y)^{2}+2(1+x / y) \ln (x / y) \ln (1+x / y) \\
& +\frac{\left(24+192 x / y-317(x / y)^{2}\right)}{24(x / y)} \ln (x / y)+\frac{\left(1-3 x / y-27(x / y)^{2}+29(x / y)^{3}\right)}{3(x / y)^{2}} \ln (1-x / y) \\
& \left.+\frac{\left(-8+24 x / y+510(x / y)^{2}-517(x / y)^{3}\right)}{72(x / y)^{2}}\right)-16 C_{F}(x / y)^{2}\left(\frac{5+12(x / y)^{2}}{30} \ln (x / y)^{2}\right. \\
& -(1-x / y) \ln (1-x / y)+\frac{\left(-2+10(x / y)^{3}-12(x / y)^{5}\right)}{15(x / y)^{3}}(+\operatorname{dilog}(1+x / y) \\
& +\ln (x / y) \ln (1+x / y))+2 \frac{5-6(x / y)^{2}}{15} P i^{2} / 6+\frac{4-2 x / y-27(x / y)^{2}-6(x / y)^{3}}{30(x / y)^{2}} \ln (x / y) \\
& \left.\left.+\frac{(1-x / y)\left(-4-18 x / y+105(x / y)^{2}\right)}{30(x / y)^{2}}\right)\right]
\end{aligned}
$$

For the $\mathrm{SU}(\mathrm{N})$ gauge group, we have $C_{A}=N, C_{F}=$ $\left(N^{2}-1\right) / 2 N, T_{F}=N_{f} T_{R}$, and $T_{R}=1 / 2$ where $C_{F}$ and $C_{A}$ are the color Cassimir operators.

\section{REFERENCES}

1.Yu.L.Dokshitzer, Sov.Phys.JETP 46, 641(1977); G.Altarelli and G.Parisi, Nucl.Phys.B 126, 298(1977); V.N.Gribov and L.N.Lipatov, Sov.J.Nucl.Phys. 15, 438(1972).

2.A.M.Cooper-Sarkar et.al., Z.Phys.C39, 281(1988); Acta.Phys.Polon.B34, 2911(2003).

3. J.L.Miramontes, J.sanchez Guillen and E.Zas, Phys.Rev.D 35, 863(1987).

4. D.I.Kazakov, et.al., Phys.Rev.Lett. 65, 1535(1990). 5.M.M.Block, et.al., Phys.Rev.D77,094003(2008).
6. A.Donnachie and P.V.Landshoff, Phys.Lett.B 437, 408(1998).

7. A.Donnachie and P.V.Landshoff, Phys.Lett.B 550, 160(2002)

; P.V.Landshoff hep-ph/0203084.

8. M.Gluk, E.Reya and A.Vogt, Z.Phys.C 67, 433(1995 ); Euro.Phys.J.C 5, 461(1998).

9.F.D. Aaron et al. [H1 Collaboration], phys.Lett.B665, 139(2008); Eur.Phys.J.C71,1579(2011). C.Adloff et al. [H1 Collaboration], Eur.Phys.J.C21, 33(2001).

10. S.Moch, J.A.M.Vermaseren, A.vogt, Phys.Lett.B 606, 123(2005); A. Vogt, S. Moch and J. A. M. Vermaseren, Nucl. Phys. B691, 129(2004).

11. A. D. Martin, R. G. Roberts, W. J. Stirling and R. Thorne, Phys. Lett. B531, 216(2001). 


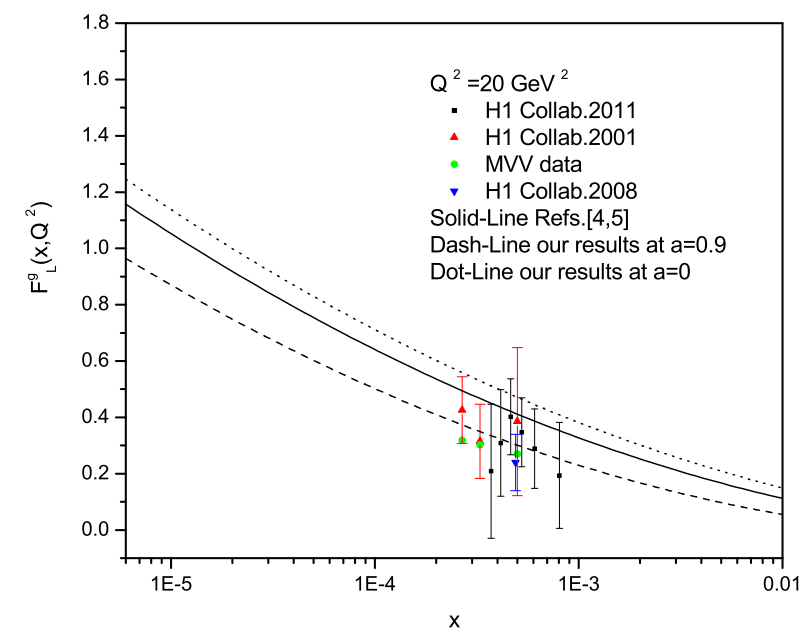

FIG. 1: LO longitudinal structure function at $Q^{2}=20 \mathrm{GeV}^{2}$ according to the gluon distribution input [5] compared with results Ref.[2], H1 data [9] and MVV [10] prediction. Our results at the point of expansion $a=0$ shown with dot-line and at the point of expansion $a=0.9$ shown with dash-line.

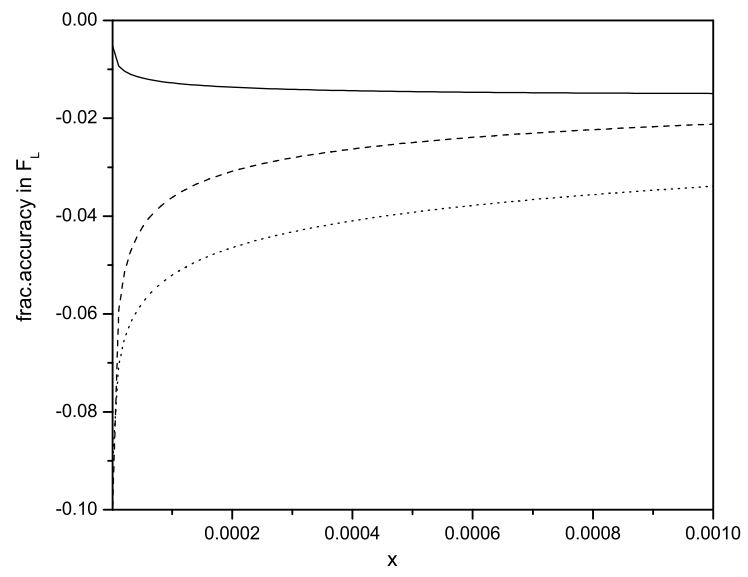

FIG. 2: Fractional accuracy plots for $F_{L}$ using input gluon distributions [5-8] with respect to the NLO QCD parameterizations of MRST [11] for $Q^{2}=20 \mathrm{GeV}^{2}$. The solid $\left(F_{\text {Block,MRST }}\right)$, dash $\left(F_{D L, M R S T}\right)$ and $\operatorname{dot}\left(F_{G R V, M R S T}\right)$ curves are taken from the Block model, DL model and GRV parameterization respectively. 


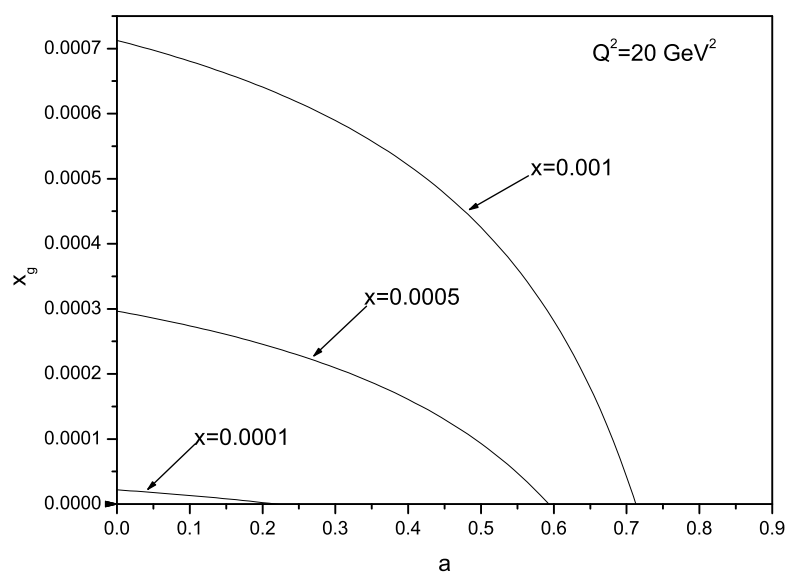

FIG. 3: Behaviour of $x_{g}\left(=k x, k=\frac{1}{1-a}\left(1-a+\frac{B}{A}\right)\right)$ vs the points of expansion $a$ at NLO.

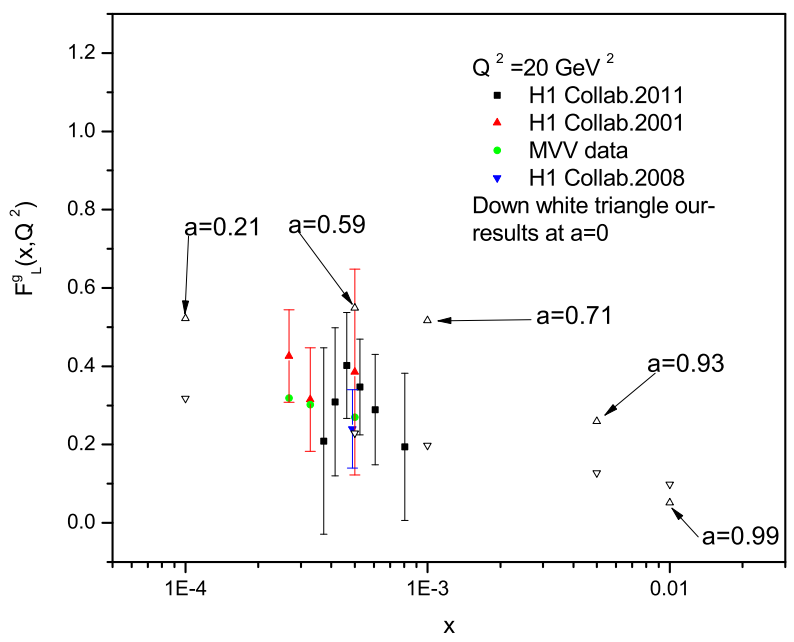

FIG. 4: NLO longitudinal structure function at $Q^{2}=20 \mathrm{GeV}^{2}$ according to the gluon distribution input [5] compared with results Ref.[2], H1 data [9] and MVV [10] prediction. Our results at the point of expansion $a=0$ shown with downwhite triangle and at the maximum point of expansion shown with up- white triangle. 


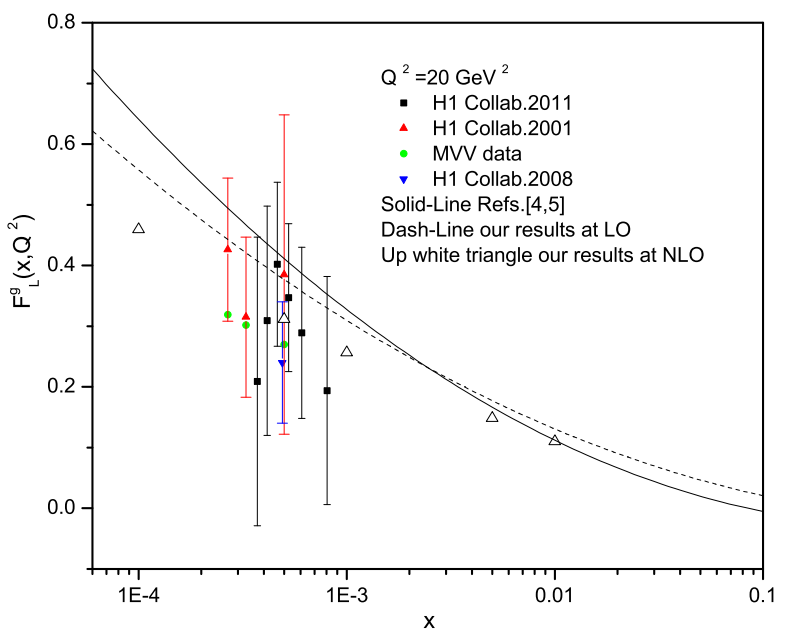

FIG. 5: Behaviour of the LO and NLO longitudinal structure function at $Q^{2}=20 \mathrm{GeV}^{2}$ according to the gluon distribution input [5] compared with results Ref.[2](solid- line), H1 data [9] and MVV [10] prediction. Our results according to the hard-Pomeron behaviour at LO shown with dash-line and at NLO shown with up- white triangle. 